\title{
Association of KCTD10, MVK, and MMAB polymorphisms with dyslipidemia and coronary heart disease in Han Chinese population
}

Jie Sun ${ }^{1 \dagger}$, Yun Qian ${ }^{2+}$, Yue Jiang ${ }^{1}$, Jiaping Chen ${ }^{1}$, Juncheng Dai ${ }^{1}$, Guangfu Jin ${ }^{1}$, Jianming Wang ${ }^{1,3}$, Zhibin Hu', Sijun Liu ${ }^{1,3^{*}}$, Chong Shen ${ }^{1^{*}}$ and Hongbing Shen ${ }^{1}$

\begin{abstract}
Background: Several genome-wide association studies have discovered novel loci at chromosome 12q24, which includes mevalonate kinase (MVK), methylmalonic aciduria (cobalamin deficiency) cblB type (MMAB), and potassium channel tetramerization domain-containing 10 (KCTD10), all of which influence HDL-cholesterol concentrations. However, there are few reports on the associations between these polymorphisms and HDL-C concentrations in Chinese population. This study aimed to evaluate the associations between functional polymorphisms in three genes (MVK, MMAB and KCTD10) and HDL-C concentrations, as well as coronary heart disease (CHD) susceptibility in Chinese individuals.
\end{abstract}

Methods: We systematically selected and genotyped 18 potentially functional polymorphisms in MVK, MMAB and KCTD10 by using the TaqMan OpenArray Genotyping System in a Chinese population including 399 dyslipidemia cases, 697 CHD cases and 465 controls. Multivariate logistic regression analyses were performed to estimate the relationship between the genotypes and dyslipidemia, CHD risk with adjustment of relevant confounders.

Results: Among six polymorphisms showing significant associations with dyslipidemia, the minor alleles of rs11066782 in KCTD10, rs11613718 in KCTD10 and rs11067233 in MMAB were significantly associated with a decreased risk of CHD (additive model: $\mathrm{OR}=0.71,95 \% \mathrm{Cl}=0.53-0.97, P=0.029$ for $\mathrm{rs} 11066782 ; \mathrm{OR}=0.73,95 \% \mathrm{Cl}=0.54-0.99, P=0.044$ for $\mathrm{rs} 11613718$ and $\mathrm{OR}=0.57,95 \% \mathrm{Cl}=0.40-0.80, P=0.001$ for rs11067233). Further combined analysis showed that individuals carrying "3-4" favorable alleles presented a $62 \%(\mathrm{OR}=0.38,95 \% \mathrm{Cl}=0.21-0.66)$ decreased risk of CHD compared with those carrying "0-2" favorable alleles.

Conclusions: These findings suggest that rs11066782 in KCTD10, rs11613718 in KCTD10 and rs11067233 in MMAB may contribute to the susceptibility of CHD by altering plasma HDL-C levels in Han Chinese.

Keywords: MVK, MMAB, KCTD10, HDL-C, Coronary heart disease

\footnotetext{
* Correspondence: sjliu@njmu.edu.cn; sc100@126.com

${ }^{\dagger}$ Equal contributors

${ }^{1}$ Department of Epidemiology, School of Public Health, Nanjing Medical

University, 101 Longmian AV., Nanjing, Jiangsu 211166, China

Full list of author information is available at the end of the article
} 


\section{Background}

Coronary heart disease (CHD) is one of the leading causes of morbidity and mortality in the world [1]. In China, it is reported that more than 700,000 people die from CHD each year [2]. CHD is a complex and multifactorial disorder caused by various environmental and genetic factors $[3,4]$. Clinical and epidemiological studies have demonstrated that the presence of low levels of high-density lipoprotein-cholesterol (HDL-C) in plasma increases the risk of developing $\mathrm{CHD}$ [5-8]. The mechanism by which HDL-C confers protection against atherosclerosis includes reverse cholesterol transport from peripheral tissues to the liver [9], inhibition of low-density lipoprotein-cholesterol (LDL-C) oxidation, and stabilization of the production of prostacyclin [10]. While smoking, diet and physical activity have a role in determining plasma HDL-C concentrations, family and twin studies have shown that about half of the variation in this trait is genetically determined [11, 12]. In 2008, a genome-wide association study (GWAS) conducted in populations of European descent discovered novel loci at chromosome 12q24, which includes mevalonate kinase $(M V K)$, methylmalonic aciduria (cobalamin deficiency) cbIB type $(M M A B)$, and potassium channel tetramerization domain-containing 10 (KCTD10), all of which influence HDL-C concentrations [13]. This association has been consistently replicated in the subsequent studies [14-16]. MVK encodes the mevalonate kinase, which catalyzes an early step in the biosynthesis of cholesterol [17]. $M M A B$ encodes the enzyme which catalyzes the formation of adenosylcobalamin, a critical factor for degradation of cholesterol [17]. KCTD10, which is close to $M V K$ and $M M A B$ genes, has shown to contribute to the susceptibility of obesity, diabetes and atherosclerosis [18]. Therefore, $M V K, M M A B$ and KCTD10 genes may be candidates as susceptibility genes modulating HDL-C concentrations and then affect the risk of dyslipidemia and $\mathrm{CHD}$.

To determine whether polymorphisms in $M V K, M M A B$ and $K C T D 10$ are independently associated with the risk of dyslipidemia and CHD, we conducted a case-control study with 399 dyslipidemia cases and 465 controls in Han Chinese. In addition, for those loci showing significant associations with dyslipidemia, we further evaluated the associations between these polymorphisms and CHD risk including $697 \mathrm{CHD}$ cases and 465 controls.

\section{Methods}

\section{Study population}

In this study, we performed a two-stage case-control study. The first-stage analysis was designed to discover the suggestive variants associated with dyslipidemia in a Chinese population consisting of 399 cases and 465 controls from a community based cohort study of noninfectious diseases in Changzhou and Nantong cities,
Jiangsu Province, China. In this study, eligible subjects aged over 30 years old were enrolled in 2004 and 2007, and the baseline information including demographic, disease history and risk factors for chronic disease was obtained and a detailed clinical examination was conducted. Physical examinations, including measurements of height, weight and blood pressure as well as laboratory tests to measure total cholesterol (TC), triglycerides (TG), HDL-C and fasting plasma glucose concentrations, were performed for each subject. Fasting blood samples for routine laboratory examinations were obtained early in the morning after an overnight fast. All biochemical parameters were measured enzymatically on an auto-analyzer (Hitachi 7180 Biochemistry Auto-analyzer, Japan) according to the manufacturer's instructions.

According to the Guidelines on Prevention and Treatment of dyslipidemia in Chinese Adults [19], 399 subjects with $\mathrm{HDL}-\mathrm{C}<1.04 \mathrm{mmol} / \mathrm{L}$ were defined as cases with dyslipidemia (Low-HDL cholesterolemia), while 465 subjects not meeting this criteria were randomly selected and matched with the cases for age and sex. Subjects were excluded from the study if they had a history of diabetes, coronary heart disease or cancer, or those who were taking lipid-lowering medications.

The second-stage analysis was to determine whether the loci that influence the HDL-C levels in the first-stage also have an effect on CHD susceptibility, which consisted of 697 CHD cases from People's Hospital of Yixing City, Jiangsu Province, China and 465 controls defined in the first-stage. In brief, CHD cases were defined as having angiographic coronary stenosis with $\geq 50 \%$ lumen reduction in at least one major epicardial coronary artery. All patients were genetically unrelated ethnic Han Chinese from Yixing city. After informed consent was obtained, the information of demographic characteristics, risk factors for $\mathrm{CHD}$, history of vascular events and clinical diagnosis were written from the clinical records. A 5-ml venous blood sample was collected from each patient.

\section{SNP selection}

Based on the public HapMap SNP database (phase II + III Feb 09, on NCBI B36 assembly, dbSNP b126) and the HaploView 4.2 software, common SNPs (MAF $\geq 0.05$ ) in the three genes ( $M V K, M M A B$ and $K C T D 10)$ were screened in gene regions (including 2-kb up-stream region of each gene) in Chinese Han population. A total of 21 potentially functional SNPs were selected after the prediction by using SNPinfo Web Server (http://snpinfo. niehs.nih.gov/) which is a comprehensive web-based tool designed to select SNPs based on GWAS results, linkage disequilibrium, and predict potential functional characteristics of polymorphisms. Besides, in order to validate the findings of GWAS [13, 15], rs2338104 and rs7134594 were also included. However, three SNPs were excluded 
for the failed probe design and two SNPs were removed due to genotyping failure (call rates $<95 \%$ ). As a result, 18 loci were finally included in the current study. The linkage disequilibrium (LD) association for the 18 loci was further analyzed (Additional file 1: Figure S1).

\section{Genotyping}

Genomic DNA was isolated from leucocytes of venous blood by proteinase $\mathrm{K}$ digestion and phenol/chloroform extraction. All SNPs were genotyped by using the TaqMan OpenArray Genotyping System (Applied Biosystems lnc, USA). Fluorescence-based polymerase chain reaction (PCR) reagents were used to provide qualitative detection of targets using post-PCR (endpoint) analysis. A total volume of $5 \mu \mathrm{l}$ with $2.5 \mu \mathrm{l}$ TaqMan OpenArray Master Mix and $2.5 \mu \mathrm{l}$ normalized human DNA sample (50 $\mathrm{ng} / \mu \mathrm{l}$ ) were loaded and amplified on customized arrays following the manufacturer's instructions. Each 48-sample array chip contained two NTCs (no template controls). All 18 SNPs were successfully genotyped with call rates $>95 \%$. Additionally, $10 \%$ of samples were randomly selected for genotyping in duplicates and the results demonstrated a high degree of concordance (>99\%) among the duplicate pairs.

\section{Statistical analysis}

Data were shown as mean \pm standard deviation (SD) or $\mathrm{n}$ (\%). Differences in the distributions of demographic characteristics, selected variables and genotypes frequencies between cases and controls were analyzed by $\chi^{2}$ test or Student $t$ test. Hardy-Weinberg equilibrium was tested by a goodness-of-fit $\chi^{2}$ test to compare the observed genotype frequencies to expected frequencies among the control subjects. Associations between the genotypes and dyslipidemia, CHD risk were estimated by computing the odds ratios (OR) and $95 \%$ confidence intervals (CIs) from logistic regression analyses with adjustment for age, sex, smoking status and body mass index (BMI). The Chi-square-based $\mathrm{Q}$ test was used to test the heterogeneity of associations between subgroups. The effects of selected loci on plasma TC, TG and HDL-C concentrations were evaluated using the multiple linear regression method with adjustment for age, sex, smoking status and BMI. The significance level was set at $P<0.05$ and $P$ values were given for two-sided tests. All statistical analyses were performed using R software (Version 3.0.2, 2013-09-25; R Foundation for Statistical Computing, http://www.cran.r-project.org/). Data in this study was available (Additional file 2).

\section{Results}

\section{Characteristics of the subjects}

The characteristics of the subjects are shown in Table 1 . There were no statistically significant differences between
Table 1 Characteristics of the subjects

\begin{tabular}{llll}
\hline Variables & Controls & $\begin{array}{l}\text { Cases with } \\
\text { dyslipidemia } \\
(N=399)\end{array}$ & $\begin{array}{l}\text { Cases with coronary } \\
\text { heart disease } \\
(N=697)\end{array}$ \\
\hline Age (years) & $49.48 \pm 12.46$ & $48.97 \pm 12.68$ & $67.61 \pm 11.13$ \\
BMl $\left(\mathrm{kg} / \mathrm{m}^{2}\right)$ & $21.98 \pm 3.01$ & $25.34 \pm 3.23$ & \\
Sex & & & \\
$\quad$ Male & $216(46.45)$ & $193(48.37)$ & $404(57.96)$ \\
Female & $249(53.55)$ & $206(51.63)$ & $293(42.04)$ \\
Smoking & & & \\
$\quad$ Ever & $148(31.83)$ & $131(33.68)$ & $185(26.54)$ \\
$\quad$ Never & $317(68.17)$ & $258(66.32)$ & $512(73.46)$ \\
TC $(\mathrm{mmol} / \mathrm{L})$ & $3.94 \pm 0.60$ & $4.37 \pm 0.92$ & $4.48 \pm 2.05$ \\
TG $(\mathrm{mmol} / \mathrm{L})$ & $0.75 \pm 0.22$ & $3.16 \pm 1.33$ & $1.61 \pm 1.48$ \\
HDL-C (mmol/L) & $1.81 \pm 0.31$ & $0.81 \pm 0.18$ & $1.18 \pm 0.31$ \\
\hline Data were shown & & &
\end{tabular}

Data were shown as mean \pm standard deviation (SD) or $n(\%)$ $B M I$ body mass index, $T C$ total cholesterol, $T G$ triglycerides, HDL-C high-density lipoprotein-cholesterol

the cases with dyslipidemia and the controls in terms of age, sex, and smoking status. BMI $(P<0.0001)$, TC $(P<0.0001)$ and TG $(P<0.0001)$ were significantly higher in cases with dyslipidemia compared with the controls. Mean HDL-C concentration in cases with dyslipidemia $(0.81 \pm 0.18 \mathrm{mmol} / \mathrm{L})$ was significantly lower than that in controls $(1.81 \pm 0.31 \mathrm{mmol} / \mathrm{L})$. Additionally, the mean age of the CHD cases $(67.61 \pm 11.13$ years) were significantly higher than that of controls (49.48 \pm 12.46 years). The proportion of male in the CHD cases (57.96\%) was higher as compared with those in controls $(46.45 \%)(P=0.0001)$, whereas similar distributions of smokers were observed between groups $(P=0.051)$. Besides, TC $(P<0.0001)$ and TG $(P<0.0001)$ were significantly higher in the CHD cases than that in controls. HDL-C $(P<0.0001)$ concentration was significantly lower in the $\mathrm{CHD}$ cases compared with the controls.

\section{Association analyses for dyslipidemia}

The association results of 17 SNPs in codominant and additive models were described in Table 2. The SNP rs4499061 deviated from Hardy-Weinberg equilibrium among controls $(P<0.05)$ and was excluded from subsequent analyses. After adjustment of age, sex, smoking status and BMI, five polymorphisms (KCTD10 rs1477117, KCTD10 rs11066782, KCTD10 rs11613718, KCTD10 rs11615336 and MMAB rs12817689) showed significant associations with dyslipidemia risk in the codominant model (minor homozygote vs. major homozygote) and $M M A B$ rs11067233 was significantly associated with dyslipidemia risk in the additive model.

\section{Association analyses for plasma lipid concentrations}

We examined the effect of these six loci on plasma TC, TG, or HDL-C concentrations, respectively, using a linear 
Table 2 Summary results of associations between 17 potentially functional SNPs in three genes (KCTD10, MVK and MMAB) and risk of dyslipidemia

\begin{tabular}{|c|c|c|c|c|c|c|c|c|c|}
\hline \multirow[t]{2}{*}{ Gene } & \multirow[t]{2}{*}{ SNP } & \multirow[t]{2}{*}{ Allele $^{a}$} & \multirow{2}{*}{$\begin{array}{l}\text { Cases }^{\mathrm{b}} \\
(\mathrm{N}=399)\end{array}$} & \multirow{2}{*}{$\begin{array}{l}\text { Controls }^{b} \\
(N=465)\end{array}$} & \multirow{2}{*}{$\begin{array}{l}\text { MAF } \\
\text { (Cases/controls) }\end{array}$} & \multirow[t]{2}{*}{$H W E^{C}$} & \multicolumn{2}{|c|}{ Codominant model $^{d}$} & \multirow{2}{*}{$\begin{array}{l}\text { Additive model } \\
P_{\text {add }}\end{array}$} \\
\hline & & & & & & & $P_{\text {het }}$ & $P_{\text {hom }}$ & \\
\hline \multirow[t]{6}{*}{ KCTD10 } & rs1477117 & $\mathrm{G} / \mathrm{A}$ & 279/115/1 & $326 / 123 / 10$ & $0.148 / 0.156$ & 0.686 & 0.349 & 0.025 & 0.791 \\
\hline & rs11066782 & $C / T$ & 283/113/1 & $311 / 135 / 11$ & $0.145 / 0.172$ & 0.414 & 0.929 & 0.014 & 0.174 \\
\hline & rs11613718 & $C / T$ & $272 / 120 / 1$ & $308 / 139 / 9$ & $0.155 / 0.172$ & 0.138 & 0.762 & 0.038 & 0.521 \\
\hline & rs11615336 & $\mathrm{C} / \mathrm{A}$ & 282/101/1 & $317 / 120 / 10$ & $0.134 / 0.157$ & 0.730 & 0.931 & 0.024 & 0.288 \\
\hline & rs7295954 & $C / T$ & $322 / 60 / 1$ & $381 / 69 / 0$ & $0.081 / 0.077$ & 0.078 & 0.399 & - & 0.323 \\
\hline & rs1045582 & $\mathrm{G} / \mathrm{T}$ & $284 / 104 / 2$ & $321 / 124 / 6$ & $0.138 / 0.151$ & 0.118 & 0.888 & 0.112 & 0.459 \\
\hline \multirow[t]{2}{*}{ MVK } & rs3759387 & $\mathrm{G} / \mathrm{T}$ & $297 / 97 / 3$ & $330 / 126 / 7$ & $0.130 / 0.151$ & 0.194 & 0.990 & 0.303 & 0.687 \\
\hline & rs2287218 & $C / T$ & $279 / 112 / 4$ & $324 / 122 / 10$ & $0.152 / 0.156$ & 0.707 & 0.494 & 0.095 & 0.878 \\
\hline \multirow[t]{9}{*}{$M M A B$} & rs12817689 & $A / G$ & $284 / 105 / 3$ & $319 / 113 / 10$ & $0.142 / 0.150$ & 0.998 & 0.614 & 0.047 & 0.635 \\
\hline & rs2241201 & $C / G$ & 238/138/11 & $288 / 148 / 21$ & $0.207 / 0.208$ & 0.722 & 0.358 & 0.268 & 0.928 \\
\hline & rs877710 & $C / G$ & $194 / 177 / 26$ & 219/201/40 & $0.288 / 0.305$ & 0.522 & 0.656 & 0.330 & 0.714 \\
\hline & rs11067233 & $C / G$ & $315 / 74 / 2$ & $329 / 118 / 8$ & $0.100 / 0.147$ & 0.486 & 0.072 & 0.145 & 0.026 \\
\hline & rs9593 & $A / T$ & $187 / 162 / 26$ & 212/196/39 & $0.285 / 0.306$ & 0.506 & 0.958 & 0.333 & 0.539 \\
\hline & rs11067227 & $C / T$ & $316 / 67 / 2$ & $333 / 104 / 7$ & $0.092 / 0.133$ & 0.729 & 0.116 & 0.295 & 0.065 \\
\hline & rs7134594 & $C / T$ & $187 / 178 / 26$ & 213/196/40 & $0.294 / 0.307$ & 0.286 & 0.786 & 0.364 & 0.665 \\
\hline & rs11831226 & $\mathrm{A} / \mathrm{C}$ & $320 / 58 / 2$ & $391 / 71 / 0$ & $0.082 / 0.077$ & 0.074 & 0.491 & - & 0.342 \\
\hline & rs 8228 & $\mathrm{~A} / \mathrm{G}$ & $322 / 63 / 0$ & $380 / 69 / 0$ & $0.082 / 0.077$ & 0.078 & 0.349 & - & 0.349 \\
\hline
\end{tabular}

MAF minor allele frequency

${ }^{a}$ Major/minor allele

${ }^{\mathrm{b}}$ Major homozygote/heterozygote/rare homozygote

${ }^{\mathrm{c}}$ Hardy-Weinberg equilibrium test among controls

${ }^{d}$ Logistic regression with adjustment for sex, age, smoking and BMI were used to test associations in codominant $(P$ het: heterozygote vs. major homozygote;

$P$ hom: minor homozygote vs. major homozygote) and additive ( $P_{\text {add }}$ : minor homozygote vs. heterozygote vs. major homozygote) models

regression model with adjustment for age, sex, smoking status and BMI (Table 3). We found significant associations between rs11066782, rs11613718, rs11615336 and rs12817689 and TC (codominant model: $P=0.035$, $0.025,0.005$ and 0.038 , respectively), between rs1477117, rs11066782 and rs11615336 and TG (recessive model: $P=$ $0.028,0.015$ and 0.031 , respectively), between rs1477117, rs11066782, rs11613718 and rs11615336 and HDL-C (recessive model: $P=0.024,0.007,0.031$ and 0.025 , respectively), between rs11067233 and HDL-C (dominant model: $P=0.047$ ). Additionally, the minor alleles of these associated SNPs were consistently associated with lower TC or TG levels and higher HDL-C levels.

\section{Association analyses for CHD}

To further evaluate the associations of the significant polymorphisms and CHD risk, we genotyped these six promising SNPs in another 697 CHD cases. As shown in Table 4, after adjustment of age, sex and smoking status, the minor alleles of rs11066782 in KCTD10, rs11613718 in $K C T D 10$ and rs11067233 in $M M A B$ were significantly associated with a decreased risk of CHD (additive model: $\mathrm{OR}=0.71,95 \% \mathrm{CI}=0.53-0.97, P=0.029$ for rs11066782; $\mathrm{OR}=0.73,95 \% \mathrm{CI}=0.54-0.99, P=0.044$ for $\mathrm{rs} 11613718$ and $\mathrm{OR}=0.57,95 \% \mathrm{CI}=0.40-0.80, P=$ 0.001 for rs11067233).

Combined analyses were conducted to evaluate the cumulative effect of the three significant loci (Table 5). Intriguingly, we found a significant allele-dosage association between the number of favorable alleles and CHD risk $\left(P_{\text {trend }}<0.001\right)$. Compared with individuals carrying no favorable allele, those who carried " $1-2$ " and "3-4" favorable alleles had lower risk of CHD with adjusted ORs of 0.71 (0.50-0.99) and 0.33 (0.18-0.58), respectively. Individuals carrying "3-4" favorable alleles had a $62 \%(\mathrm{OR}=0.38$, $95 \% \mathrm{CI}=0.21-0.66)$ decreased risk of CHD compared with those carrying " $0-2$ " favorable alleles.

We also performed stratification analyses for the effect of rs11066782, rs11613718 and rs11067233 based on age, sex and smoking status (Additional file 3: Table S1). In $\geq 48$ years group, rs11066782, rs11613718 and rs11067233 were significantly associated with a decreased risk of CHD after adjustment for the other covariates $(\mathrm{OR}=0.70,95 \%$ $\mathrm{CI}=0.50-0.97 ; \mathrm{OR}=0.71,95 \% \mathrm{CI}=0.51-0.99$ and $\mathrm{OR}=$ 0.55 , $95 \% \mathrm{CI}=0.39-0.79$, respectively). Sex stratification analysis indicated that rs11613718 had statistically associated with $\mathrm{CHD}$ in male $(\mathrm{OR}=0.61,95 \% \mathrm{CI}=0.38-0.98)$ while rs11067233 had statistically associated with CHD in 
Table 3 Associations between selected single nucleotide polymorphisms and plasma lipid concentrations

\begin{tabular}{|c|c|c|c|c|c|c|}
\hline Polymorphisms & $\mathrm{TC}(\mathrm{mmol} / \mathrm{L})$ & $P^{a}$ & TG (mmol/L) & $P^{a}$ & $\mathrm{HDL}-\mathrm{C}(\mathrm{mmol} / \mathrm{L})$ & $P^{a}$ \\
\hline \multicolumn{7}{|l|}{ rs $1477117(G>A)$} \\
\hline $\mathrm{GG}(n=605)$ & $4.17 \pm 0.80$ & 0.143 & $1.86 \pm 1.49$ & 0.848 & $1.34 \pm 0.55$ & 0.456 \\
\hline GA $(n=238)$ & $4.11 \pm 0.80$ & & $1.91 \pm 1.58$ & & $1.35 \pm 0.59$ & \\
\hline $\mathrm{AA}(n=11)$ & $3.68 \pm 0.63$ & & $0.94 \pm 0.50$ & & $1.68 \pm 0.50$ & \\
\hline $\mathrm{GG}(n=605)$ & $4.17 \pm 0.80$ & 0.255 & $1.86 \pm 1.49$ & 0.733 & $1.34 \pm 0.55$ & 0.812 \\
\hline $\mathrm{GA}+\mathrm{AA}(n=249)$ & $4.09 \pm 0.80$ & & $1.87 \pm 1.56$ & & $1.36 \pm 0.59$ & \\
\hline $\mathrm{GG}+\mathrm{GA}(n=843)$ & $4.15 \pm 0.80$ & 0.081 & $1.88 \pm 1.52$ & 0.028 & $1.34 \pm 0.56$ & 0.024 \\
\hline $\mathrm{AA}(n=11)$ & $3.68 \pm 0.63$ & & $0.94 \pm 0.50$ & & $1.68 \pm 0.50$ & \\
\hline \multicolumn{7}{|l|}{ rs11066782 (C > T) } \\
\hline CC $(n=594)$ & $4.19 \pm 0.80$ & 0.035 & $1.90 \pm 1.50$ & 0.319 & $1.33 \pm 0.55$ & 0.065 \\
\hline$C T(n=248)$ & $4.07 \pm 0.79$ & & $1.85 \pm 1.58$ & & $1.38 \pm 0.60$ & \\
\hline$\Pi(n=12)$ & $3.82 \pm 0.77$ & & $0.93 \pm 0.48$ & & $1.71 \pm 0.49$ & \\
\hline CC $(n=594)$ & $4.19 \pm 0.80$ & 0.052 & $1.90 \pm 1.50$ & 0.653 & $1.33 \pm 0.55$ & 0.197 \\
\hline$C T+\Pi(n=260)$ & $4.06 \pm 0.79$ & & $1.80 \pm 1.56$ & & $1.39 \pm 0.59$ & \\
\hline$C C+C T(n=842)$ & $4.15 \pm 0.80$ & 0.202 & $1.89 \pm 1.52$ & 0.015 & $1.34 \pm 0.56$ & 0.007 \\
\hline$\Pi(n=12)$ & $3.82 \pm 0.77$ & & $0.93 \pm 0.48$ & & $1.71 \pm 0.49$ & \\
\hline \multicolumn{7}{|l|}{ rs11613718 (C>T) } \\
\hline$C C(n=580)$ & $4.18 \pm 0.79$ & 0.025 & $1.88 \pm 1.50$ & 0.599 & $1.33 \pm 0.55$ & 0.243 \\
\hline $\mathrm{CT}(n=259)$ & $4.07 \pm 0.78$ & & $1.86 \pm 1.56$ & & $1.37 \pm 0.59$ & \\
\hline$\Pi(n=10)$ & $3.71 \pm 0.66$ & & $0.96 \pm 0.52$ & & $1.70 \pm 0.53$ & \\
\hline CC $(n=580)$ & $4.18 \pm 0.79$ & 0.042 & $1.88 \pm 1.50$ & 0.914 & $1.33 \pm 0.55$ & 0.461 \\
\hline$C T+\Pi(n=269)$ & $4.05 \pm 0.78$ & & $1.83 \pm 1.55$ & & $1.38 \pm 0.59$ & \\
\hline$C C+C T(n=839)$ & $4.15 \pm 0.79$ & 0.131 & $1.88 \pm 1.52$ & 0.053 & $1.34 \pm 0.56$ & 0.031 \\
\hline$\Pi(n=10)$ & $3.71 \pm 0.66$ & & $0.96 \pm 0.52$ & & $1.70 \pm 0.53$ & \\
\hline \multicolumn{7}{|l|}{ rs11615336 (C > A) } \\
\hline$C C(n=599)$ & $4.19 \pm 0.80$ & 0.005 & $1.90 \pm 1.51$ & 0.191 & $1.34 \pm 0.55$ & 0.275 \\
\hline $\mathrm{CA}(n=221)$ & $4.03 \pm 0.75$ & & $1.80 \pm 1.53$ & & $1.36 \pm 0.60$ & \\
\hline $\mathrm{AA}(n=11)$ & $3.68 \pm 0.63$ & & $0.94 \pm 0.50$ & & $1.68 \pm 0.50$ & \\
\hline CC $(n=599)$ & $4.19 \pm 0.80$ & 0.009 & $1.90 \pm 1.51$ & 0.392 & $1.34 \pm 0.55$ & 0.546 \\
\hline $\mathrm{CA}+\mathrm{AA}(n=232)$ & $4.02 \pm 0.75$ & & $1.76 \pm 1.51$ & & $1.38 \pm 0.60$ & \\
\hline$C C+C A(n=820)$ & $4.15 \pm 0.79$ & 0.082 & $1.87 \pm 1.51$ & 0.031 & $1.34 \pm 0.56$ & 0.025 \\
\hline $\mathrm{AA}(n=11)$ & $3.68 \pm 0.63$ & & $0.94 \pm 0.50$ & & $1.68 \pm 0.50$ & \\
\hline \multicolumn{7}{|l|}{ rs12817689 (A > G) } \\
\hline $\mathrm{AA}(n=603)$ & $4.18 \pm 0.80$ & 0.038 & $1.89 \pm 1.50$ & 0.733 & $1.34 \pm 0.55$ & 0.423 \\
\hline AG $(n=218)$ & $4.06 \pm 0.78$ & & $1.90 \pm 1.58$ & & $1.35 \pm 0.60$ & \\
\hline $\mathrm{GG}(n=13)$ & $3.88 \pm 0.67$ & & $1.34 \pm 1.20$ & & $1.54 \pm 0.57$ & \\
\hline $\mathrm{AA}(n=603)$ & $4.18 \pm 0.80$ & 0.051 & $1.89 \pm 1.50$ & 0.995 & $1.34 \pm 0.55$ & 0.669 \\
\hline $\mathrm{AG}+\mathrm{GG}(n=231)$ & $4.05 \pm 0.77$ & & $1.87 \pm 1.56$ & & $1.36 \pm 0.60$ & \\
\hline $\mathrm{AA}+\mathrm{AG}(n=821)$ & $4.14 \pm 0.79$ & 0.278 & $1.89 \pm 1.52$ & 0.172 & $1.34 \pm 0.56$ & 0.099 \\
\hline $\mathrm{GG}(n=13)$ & $3.88 \pm 0.67$ & & $1.34 \pm 1.20$ & & $1.54 \pm 0.57$ & \\
\hline \multicolumn{7}{|l|}{ rs11067233 (C > G) } \\
\hline$C C(n=644)$ & $4.15 \pm 0.80$ & 0.392 & $1.94 \pm 1.54$ & 0.123 & $1.32 \pm 0.56$ & 0.049 \\
\hline CG $(n=192)$ & $4.17 \pm 0.82$ & & $1.68 \pm 1.46$ & & $1.44 \pm 0.55$ & \\
\hline $\mathrm{GG}(n=10)$ & $4.10 \pm 0.63$ & & $1.38 \pm 1.12$ & & $1.51 \pm 0.42$ & \\
\hline
\end{tabular}


Table 3 Associations between selected single nucleotide polymorphisms and plasma lipid concentrations (Continued)

\begin{tabular}{|c|c|c|c|c|c|c|}
\hline CC $(n=644)$ & $4.15 \pm 0.80$ & 0.347 & $1.94 \pm 1.54$ & 0.137 & $1.32 \pm 0.56$ & 0.047 \\
\hline $\mathrm{CG}+\mathrm{GG}(n=202)$ & $4.17 \pm 0.81$ & & $1.66 \pm 1.44$ & & $1.44 \pm 0.55$ & \\
\hline$C C+C G(n=836)$ & $4.15 \pm 0.80$ & 0.952 & $1.88 \pm 1.52$ & 0.482 & $1.35 \pm 0.56$ & 0.591 \\
\hline $\mathrm{GG}(n=10)$ & $4.10 \pm 0.63$ & & $1.38 \pm 1.12$ & & $1.51 \pm 0.42$ & \\
\hline
\end{tabular}

TC total cholesterol, TG triglycerides, HDL-C high-density lipoprotein-cholesterol

${ }^{a}$ Multiple linear regression with adjustment for sex, age, smoking and BMI

female $(\mathrm{OR}=0.35,95 \% \mathrm{CI}=0.20-0.62)$ after adjustment for covariates respectively. Besides, rs11067233 was significantly associated with a decreased risk of CHD in neversmokers after adjustment for relevant covariates $(\mathrm{OR}=$ $0.37,95 \% \mathrm{CI}=0.23-0.59$ ).

\section{Discussion}

In this study, we investigated the relationship of 17 SNPs located in three genes ( $M V K, M M A B$ and $K C T D 10)$ with HDL-C concentrations and CHD risk in a Chinese population. We found that rs11067233 in $M M A B$, rs11066782 and rs11613718 in KCTD10 were associated with HDL-C concentrations and with CHD risk.

The KCTD10 gene, located on chromosome 12q24, is a member of the polymerase delta-interacting protein 1 gene family [20]. Reports showed that KCTD10 was highly expressed in human heart, skeletal muscle, and placenta, and play a vital role in DNA synthesis by interacting with proliferating cell nuclear antigen and polymerase $\delta$ [21]. In A549 lung adenocarcinoma cells, down-regulation of KCTD10 could inhibit cell proliferation [22]. Transcription factors SP1 and AP- $2 \alpha$ could bind to the promoter region of human $K C T D 10$, and regulate its expression [23]. A recent study revealed KCTD10 as a novel prognostic biomarker in gastrointestinal stromal tumor [24]. According to Chen et al., Murine Kctd10 has been implicated in a metabolic network perturbed by loci contributing to the susceptibility of obesity, diabetes, and atherosclerosis [18]. And by extension, the involvement of human KCTD10 in this metabolic network supports its association with HDL-C concentrations and CHD development. In the present study, we found, for the first time, that the minor alleles of rs11066782 and rs11613718 in KCTD10 were associated with higher HDL-C concentrations and lower CHD risk in a Chinese population. Since high levels of HDL-C could reduce the risk of ischaemic heart disease $[8,25]$, it is reasonable that the variant genotypes of the two polymorphisms may affect CHD susceptibility by increasing HDL-C concentrations. Located at intron 1 of KCTD10 gene, the two SNPs are in high linkage disequilibrium with each other $\left(r^{2}=0.92\right)$, and both of them may affect gene expression by altering transcription factor binding sites (TFBS) of KCTD10 based on SNPinfo Web Server (http://snpinfo.niehs.nih.gov/). Analysis of Encyclopedia of DNA Elements data as implemented in online tool, RegulomeDB (http://regulomedb.org/), indicated that the two polymorphisms may influence the histone modifications and the expression of KCTD10, which may serve as regulatory variants in the development of coronary heart disease. Moreover, data from GTEx (http://www.gtexportal.org/home/) revealed that variant genotypes of the two potential SNPs were significantly associated with higher expression levels of KCTD10 in liver tissues in all HapMap subjects $(P=$ 0.041 for rs11066782 and $P=0.038$ for rs11613718, respectively; Additional file 4: Figure S2a, b). Besides, stratified analyses subsequently demonstrated that the protective effects of the variant genotypes were greater in those aged $\geq 48$ years for rs11066782 and rs11613718, and greater in men for rs11613718 compared with other individuals. Since the prevalence of CHD increases with advancing age $[26,27]$ and is greater in men than women

Table 4 Associations between selected single nucleotide polymorphisms and risk of coronary heart disease

\begin{tabular}{|c|c|c|c|c|c|c|c|c|c|c|}
\hline \multirow[t]{2}{*}{ Gene } & \multirow[t]{2}{*}{ SNP } & \multirow{2}{*}{$\begin{array}{l}\text { Cases }^{a} \\
(N=697)\end{array}$} & \multirow{2}{*}{$\begin{array}{l}\text { Controls }^{a} \\
(N=465)\end{array}$} & \multirow{2}{*}{$\begin{array}{l}\text { MAF } \\
\text { (Cases/controls) }\end{array}$} & \multicolumn{2}{|c|}{$\begin{array}{l}\text { Heterozygote vs. major } \\
\text { homozygote }\end{array}$} & \multicolumn{2}{|c|}{$\begin{array}{l}\text { Minor homozygote vs. major } \\
\text { homozygote }\end{array}$} & \multicolumn{2}{|l|}{ Additive model } \\
\hline & & & & & OR $(95 \% \mathrm{Cl})^{\mathrm{b}}$ & $p^{\mathrm{b}}$ & OR $(95 \% \mathrm{Cl})^{\mathrm{b}}$ & $p^{b}$ & OR $(95 \% \mathrm{Cl})^{\mathrm{b}}$ & $p^{b}$ \\
\hline \multirow[t]{4}{*}{ KCTD10 } & 7 & $521 / 156 / 16$ & $326 / 123 / 10$ & $0.136 / 0.156$ & $0.70(0.49-1.00)$ & 0.048 & $1.13(0.39-3.33)$ & 0.820 & $0.79(0.58-1.08)$ & 0.135 \\
\hline & rs11066782 & $517 / 160 / 15$ & $311 / 135 / 11$ & $0.137 / 0.172$ & $0.62(0.43-0.88)$ & 0.007 & $1.02(0.35-2.94)$ & 0.975 & $0.71(0.53-0.97)$ & 0.029 \\
\hline & rs11613718 & $494 / 160 / 15$ & $308 / 139 / 9$ & $0.142 / 0.172$ & $0.63(0.44-0.90)$ & 0.010 & $1.12(0.37-3.35)$ & 0.840 & $0.73(0.54-0.99)$ & 0.044 \\
\hline & rs11615336 & $502 / 166 / 16$ & $317 / 120 / 10$ & $0.145 / 0.157$ & $0.74(0.52-1.06)$ & 0.104 & $1.12(0.38-3.30)$ & 0.835 & $0.83(0.61-1.12)$ & 0.223 \\
\hline \multirow[t]{2}{*}{$M M A B$} & rs12817689 & $519 / 158 / 15$ & $319 / 113 / 10$ & $0.136 / 0.150$ & $0.78(0.54-1.12)$ & 0.173 & $1.10(0.38-3.21)$ & 0.865 & $0.85(0.62-1.16)$ & 0.311 \\
\hline & rs11067233 & $562 / 122 / 7$ & $329 / 118 / 8$ & $0.098 / 0.147$ & $0.50(0.34-0.73)$ & $<0.001$ & $0.73(0.20-2.67)$ & 0.631 & $0.57(0.40-0.80)$ & 0.001 \\
\hline
\end{tabular}

MAF minor allele frequency, $O R$ odds ratio, $\mathrm{Cl}$ confidence interval

${ }^{a}$ Major homozygote/heterozygote/rare homozygote

${ }^{b}$ Logistic regression with adjustment for sex, age and smoking 
Table 5 Combined analysis of the cumulative effect of rs11066782, rs11613718 and rs11067233 on coronary heart disease risk

\begin{tabular}{lllll}
\hline Combined genotypes $^{\mathrm{a}}$ & Cases & Controls & OR $(95 \% \mathrm{Cl})^{\mathrm{b}}$ & $P^{\mathrm{b}}$ \\
\hline 0 & 383 & 222 & 1.00 & \\
$1-2$ & 244 & 166 & $0.71(0.50-0.99)$ & 0.045 \\
$3-4$ & 33 & 55 & $0.33(0.18-0.58)$ & $<0.001$ \\
$P$ for trend & & & & $<0.001$ \\
$0-2$ & 627 & 388 & 1.00 & \\
$3-4$ & 33 & 55 & $0.38(0.21-0.66)$ & $<0.001$ \\
\hline
\end{tabular}

$O R$ odds ratio, $\mathrm{Cl}$ confidence interval

${ }^{a}$ The combined genotypes were according to favorable alleles carried (rs11066782-T, rs11613718-T and rs11067233-G were considered as favorable alleles)

${ }^{b}$ Logistic regression with adjustment for sex, age and smoking

[28], it is reasonable that such genetic effects are more evident among the older people and men. Nevertheless, larger sample size and multi-ethnic population studies are warranted to support our findings.

In humans, $M V K$ and $M M A B$ are arranged in a headto-head orientation on chromosome 12 . Through a shared common promoter, $M V K$ and $M M A B$ are both regulated by sterol-responsive element-binding protein 2 (SREBP2), which is a transcription factor that controls cholesterol homeostasis [17]. Furthermore, it is also reported that these two neighboring genes participate in metabolic pathways associated with HDL metabolism. $M V K$ encodes for mevalonate kinase, which catalyzes an early step in the biosynthesis of cholesterol [17]. Homozygosity for milder mutations in $M V K$ could cause hyperimmunoglobulinemia D syndrome (HIDS), which is characterized by fever and increased levels of immunoglobulin D and A [29, 30]. In keeping with GWAS findings [13, 14], patients with HIDS have low HDL-C concentrations. However, in our study, neither rs3759387 in $M V K$ nor rs2287218 in $M V K$ showed a consistent result with the previous studies. The effects of SNPs on dyslipidemia might differ according to different ethnic background, as the rs3759387 $\mathrm{T}$ allele and rs2287218 $\mathrm{T}$ allele were found to be more enriched in the Caucasians than in Asians; Additionally, these discrepancies may be due to differences in lifestyle across populations. Functional characterizations are warranted to determine the causal variants related to plasma lipid concentrations in Asian populations. $M M A B$ encodes the cob(I)alamin adenosyltransferase, an enzyme involved in the formation of adenosylcobalamin, necessary for degradation of cholesterol [17]. In humans, deficiency of cob(I)alamin adenosyltransferase results in methylmalonic aciduria [31], and a negative correlation between urinary methylmalonic acid and red blood cell membrane cholesterol concentrations was also found in patients with schizophrenia [32]. According to Junyent et al., homozygotes for major alleles at SNPs MMAB 3U3527G >C displayed lower HDL-C concentrations than carriers of the minor alleles when they consumed diets rich in carbohydrates [33]. Besides, Marie et al. observed significant allelic expression imbalance of $22 \%$ in $M M A B$ (transcribed SNP rs11067231) and the allele associated with lower HDL-C level displayed greater $M M A B$ transcript level. This result indicated that $M M A B$ was a likely susceptibility gene influencing HDL-C levels [34]. In addition, $M M A B$ can affect TG levels through adenosylcobalamin, methylmalonyl-CoA mutase [35] and homocysteine $[36,37]$ based on several related function researches. In this study, we found that the variant genotypes of rs11067233 were associated with higher HDL-C concentrations and lower CHD risk. According to SNPinfo Web Server, rs11067233 may affect $M M A B$ gene expression by altering microRNA binding at the 3'UTR of $M M A B$. Meanwhile, rs11067233 $\mathrm{C}>\mathrm{G}$ could result in the loss of hsa-miR-603 binding to $M M A B$ with energy change of 20.90 , as predicted by miRNASNP v2.0 (http://bioinfo.life.hust.edu.cn/ miRNASNP2/), which is a online database that predicts the effect (loss or gain of function) of miRNA-related SNPs based on miRNA expression data, GWAS information and experimental validation results. Additionally, data from GTEx project suggested that the variant genotypes of rs11067233 showed relatively lower expression levels of $M M A B$ in liver tissues compared with homozygotes, although the difference did not reach the statistical significance level $(P=0.078$; Additional file 4: Figure $S 2 \mathrm{c})$. Moreover, Fogarty et al. also found that the $C$ allele of rs11067233 demonstrated $15 \%$ higher $M M A B$ expression than the G allele in human hepatocytes [34]. Taken together, it is plausible that rs11067233 is associated with $M M A B$ gene expression, which therefore could alter serum lipid concentrations, and accordingly modify the risk of coronary artery disease. Notably, we found that rs11067233 genotypes were significantly associated with lower risk of CHD among never-smokers and females. As cigarette use is a well-established risk factor for CHD $[38,39]$ and most of the never-smokers in China are females, the protective effect of rs11067233 may be diluted by environmental risk factors if they do not have a joint effect. Anyhow, further investigations in relation to biological mechanisms may lead to important insights for these findings.

Several limitations of our study should be considered. Though we observed significantly main effects of three polymorphisms on CHD risk, only rs11067233 remained significant after Bonferroni correction for multiple comparisons $(P<0.010$, Bonferroni-adjusted), suggesting that larger well-designed studies are warranted to confirm the associations identified in our study. Secondly, the sample size was moderate with limited power to detect significant associations. Thirdly, inherent selection bias could not be completely excluded due to the unbalanced 
matching in age and sex. Finally, some other confounding factors may potentially mediate the effect of selected polymorphisms on dyslipidemia and CHD risk such as alcohol consumption, physical activity, family history of cardiovascular diseases and so on. However, we applied a rigorous epidemiological design and laboratory tests and statistically adjusted for several known risk factors to minimize potential bias.

\section{Conclusions}

In conclusion, we found that rs 11067233 in $M M A B$, rs11066782 and rs11613718 in KCTD10 were associated with higher HDL-C concentrations and lower CHD risk in a Chinese population. Further studies incorporating diverse populations and functional assays are required to validate and extend these findings.

\section{Additional files}

Additional file 1: Figure S1. The LD figure for the 18 loci included in this study. (DOCX $112 \mathrm{~kb}$ )

Additional file 2: The dataset of this article. (CSV $145 \mathrm{~kb}$ )

Additional file 3: Table S1. Stratification analysis on the associations of rs11066782, rs11613718 and rs1 1067233 with coronary heart disease risk. (DOCX $16 \mathrm{~kb}$ )

Additional file 4: Figure S2. Box plots showing gene expression levels by different variant genotypes identified from GTEx database. (DOCX $101 \mathrm{~kb}$ )

\section{Abbreviations}

BMI: Body mass index; CHD: Coronary heart disease; Cl: Confidence interval: GWAS: Genome-wide association study; HDL-C: High-density lipoproteincholesterol; LD: Linkage disequilibrium; LDL-C: Low-density lipoproteincholesterol; MAF: Minor allele frequency; OR: Odds ratio; PCR: Polymerase chain reaction; SNP: Single nucleotide polymorphism; TC: Total cholesterol; TFBS: Transcription factor binding sites; TG: Triglycerides

\section{Acknowledgements}

Not applicable.

\section{Funding}

This study was financially supported by grants from the National Natural Science Foundation of China (No. 81102180 and No. 81390543), the Priority Academic Program Development of Jiangsu Higher Education Institutions (PAPD) and Jiangsu Government Scholarship for Overseas Studies. The funders had no role in the design of the study and collection, analysis, and interpretation of data and in writing the manuscript.

\section{Availability of data and materials}

The dataset supporting the conclusions of this article is included within the article's additional file.

\section{Authors' contributions}

SL, CS and HS designed the experiments. JS, YJ and JC performed the experiments. JD and GJ analyzed the data. JS, YQ and JW wrote the main manuscript. ZH and SL revised the manuscript. All authors read and approved the final manuscript.

\section{Competing interests}

The authors declare that they have no competing interests.

\section{Consent for publication}

Not applicable.

\section{Ethics approval and consent to participate}

This study was approved by the Ethical Committee of Nanjing Medical University. Written informed consent was obtained from each participant before investigation.

\section{Author details}

'Department of Epidemiology, School of Public Health, Nanjing Medical University, 101 Longmian AV., Nanjing, Jiangsu 211166, China. ²Department of Chronic Non-communicable Disease Control, Wuxi Center for Disease Control and Prevention, Wuxi 214023, China. ${ }^{3}$ Department of Social Medicine and Health Education, School of Public Health, Nanjing Medical University, 101 Longmian AV., Nanjing, Jiangsu 211166, China.

Received: 13 May 2016 Accepted: 30 September 2016

Published online: 04 October 2016

\section{References}

1. Lopez AD, Mathers CD, Ezzati M, Jamison DT, Murray CJ. Global and regional burden of disease and risk factors, 2001: systematic analysis of population health data. Lancet. 2006;367(9524):1747-57.

2. Wang F, Xu CQ, He Q, Cai JP, Li XC, Wang D, et al. Genome-wide association identifies a susceptibility locus for coronary artery disease in the Chinese Han population. Nat Genet. 2011:43(4):345-9.

3. Consortium CAD, Deloukas $P$, Kanoni $S$, Willenborg C, Farrall M, Assimes $T L$, et al. Large-scale association analysis identifies new risk loci for coronary artery disease. Nat Genet. 2013;45(1):25-33.

4. Souiden Y, Mallouli H, Meskhi S, Chaabouni Y, Rebai A, Cheour F, et al. MnSOD and GPx1 polymorphism relationship with coronary heart disease risk and severity. Biol Res. 2016;49:22.

5. Castelli WP, Garrison RJ, Wilson PW, Abbott RD, Kalousdian S, Kannel WB. Incidence of coronary heart disease and lipoprotein cholesterol levels. The Framingham Study. JAMA. 1986;256(20):2835-8.

6. Goldbourt U, Yaari S, Medalie JH. Isolated low HDL cholesterol as a risk factor for coronary heart disease mortality. A 21-year follow-up of 8000 men. Arterioscler Thromb Vasc Biol. 1997:17(1):107-13.

7. Gotto Jr AM, Brinton EA. Assessing low levels of high-density lipoprotein cholesterol as a risk factor in coronary heart disease: a working group report and update. J Am Coll Cardiol. 2004;43(5):717-24.

8. Prospective Studies C, Lewington S, Whitlock G, Clarke R, Sherliker P, Emberson J, et al. Blood cholesterol and vascular mortality by age, sex, and blood pressure: a meta-analysis of individual data from 61 prospective studies with 55,000 vascular deaths. Lancet. 2007;370(9602):1829-39.

9. Ng DS, Wong NC, Hegele RA. HDL-is it too big to fail? Nat Rev Endocrinol. 2013;9(5):308-12.

10. Mackness B, Mackness M. The antioxidant properties of high-density lipoproteins in atherosclerosis. Panminerva Med. 2012;54(2):83-90.

11. Harrap SB, Wong ZY, Scurrah KJ, Lamantia A. Genome-wide linkage analysis of population variation in high-density lipoprotein cholesterol. Hum Genet. 2006;119(5):541-6.

12. Goode EL, Cherny SS, Christian JC, Jarvik GP, de Andrade M. Heritability of longitudinal measures of body mass index and lipid and lipoprotein levels in aging twins. Twin Res Hum Genet. 2007;10(5):703-11.

13. Willer CJ, Sanna S, Jackson AU, Scuteri A, Bonnycastle LL, Clarke R, et al. Newly identified loci that influence lipid concentrations and risk of coronary artery disease. Nat Genet. 2008;40(2):161-9.

14. Kathiresan S, Willer CJ, Peloso GM, Demissie S, Musunuru K, Schadt EE, et al. Common variants at 30 loci contribute to polygenic dyslipidemia. Nat Genet. 2009:41(1):56-65

15. Teslovich TM, Musunuru K, Smith AV, Edmondson AC, Stylianou IM, Koseki $\mathrm{M}$, et al. Biological, clinical and population relevance of 95 loci for blood lipids. Nature. 2010;466(7307):707-13.

16. Global Lipids Genetics C, Willer CJ, Schmidt EM, Sengupta S, Peloso GM, Gustafsson S, et al. Discovery and refinement of loci associated with lipid levels. Nat Genet. 2013;45(11):1274-83.

17. Holleboom AG, Vergeer M, Hovingh GK, Kastelein JJ, Kuivenhoven JA. The value of HDL genetics. Curr Opin Lipidol. 2008;19(4):385-94.

18. Chen Y, Zhu J, Lum PY, Yang X, Pinto S, MacNeil DJ, et al. Variations in DNA elucidate molecular networks that cause disease. Nature. 2008;452(7186):429-35.

19. Joint Committee for Developing Chinese guidelines on Prevention and Treatment of Dyslipidemia in Adults. [Chinese guidelines on prevention and 
treatment of dyslipidemia in adults]. Zhonghua Xin Xue Guan Bing Za Zhi. 2007;35(5):390-419.

20. He H, Tan CK, Downey KM, So AG. A tumor necrosis factor alpha- and interleukin 6-inducible protein that interacts with the small subunit of DNA polymerase delta and proliferating cell nuclear antigen. Proc Natl Acad Sci U S A. 2001;98(21):11979-84.

21. Zhou J, Ren K, Liu X, Xiong X, Hu X, Zhang J. A novel PDIP1-related protein, KCTD10, that interacts with proliferating cell nuclear antigen and DNA polymerase delta. Biochim Biophys Acta. 2005;1729(3):200-3.

22. Wang Y, Zheng Y, Luo F, Fan X, Chen J, Zhang C, et al. KCTD10 interacts with proliferating cell nuclear antigen and its down-regulation could inhibit cell proliferation. J Cell Biochem. 2009;106(3):409-13.

23. Liu R, Zhou A, Ren D, He A, Hu X, Zhang W, et al. Transcription factor specificity protein 1 (SP1) and activating protein 2alpha (AP-2alpha) regulate expression of human KCTD10 gene by binding to proximal region of promoter. FEBS J. 2009;276(4):1114-24.

24. Kubota D, Yoshida A, Tsuda H, Suehara Y, Okubo T, Saito T, et al. Gene expression network analysis of ETV1 reveals KCTD10 as a novel prognostic biomarker in gastrointestinal stromal tumor. PLoS One. 2013;8(8):e73896.

25. Law MR, Wald NJ, Rudnicka AR. Quantifying effect of statins on low density lipoprotein cholesterol, ischaemic heart disease, and stroke: systematic review and meta-analysis. BMJ. 2003;326(7404):1423.

26. Abbott RD, Curb JD, Rodriguez BL, Masaki KH, Yano K, Schatz IJ, et al. Age-related changes in risk factor effects on the incidence of coronary heart disease. Ann Epidemiol. 2002;12(3):173-81.

27. Go AS, Mozaffarian D, Roger VL, Benjamin EJ, Berry JD, Blaha MJ, et al. Heart disease and stroke statistics-2014 update: a report from the American Heart Association. Circulation. 2014;129(3):e28-292.

28. Towfighi A, Zheng L, Ovbiagele B. Sex-specific trends in midlife coronary heart disease risk and prevalence. Arch Intern Med. 2009;169(19):1762-6.

29. Haas D, Hoffmann GF. Mevalonate kinase deficiencies: from mevalonic aciduria to hyperimmunoglobulinemia D syndrome. Orphanet J Rare Dis. 2006;1:13.

30. Klasen IS, Goertz JH, van de Wiel GA, Weemaes CM, van der Meer JW, Drenth JP. Hyper-immunoglobulin A in the hyperimmunoglobulinemia D syndrome. Clin Diagn Lab Immunol. 2001;8(1):58-61.

31. Dobson CM, Wai T, Leclerc D, Kadir H, Narang M, Lerner-Ellis JP, et al. Identification of the gene responsible for the cblB complementation group of vitamin B12-dependent methylmalonic aciduria. Hum Mol Genet. 2002;11(26):3361-9.

32. Ozcan O, Ipcioglu OM, Gultepe M, Basogglu C. Altered red cell membrane compositions related to functional vitamin B(12) deficiency manifested by elevated urine methylmalonic acid concentrations in patients with schizophrenia. Ann Clin Biochem. 2008;45(Pt 1):44-9.

33. Junyent M, Parnell LD, Lai CQ, Lee YC, Smith CE, Arnett DK, et al. Novel variants at KCTD10, MVK, and MMAB genes interact with dietary carbohydrates to modulate HDL-cholesterol concentrations in the Genetics of Lipid Lowering Drugs and Diet Network Study. Am J Clin Nutr. 2009;90(3):686-94.

34. Fogarty MP, Xiao R, Prokunina-Olsson L, Scott $\sqcup$, Mohlke KL. Allelic expression imbalance at high-density lipoprotein cholesterol locus MMAB-MVK. Hum Mol Genet. 2010;19(10):1921-9.

35. Maiti N, Widjaja L, Banerjee R. Proton transfer from histidine 244 may facilitate the 1,2 rearrangement reaction in coenzyme $\mathrm{B}(12)$-dependent methylmalonyl-CoA mutase. J Biol Chem. 1999;274(46):32733-7.

36. Clarke R, Armitage J. Vitamin supplements and cardiovascular risk: review of the randomized trials of homocysteine-lowering vitamin supplements. Semin Thromb Hemost. 2000;26(3):341-8.

37. Werstuck GH, Lentz SR, Dayal S, Hossain GS, Sood SK, Shi YY, et al. Homocysteine-induced endoplasmic reticulum stress causes dysregulation of the cholesterol and triglyceride biosynthetic pathways. J Clin Invest. 2001;107(10):1263-73.

38. James RW, Leviev I, Righetti A. Smoking is associated with reduced serum paraoxonase activity and concentration in patients with coronary artery disease. Circulation. 2000;101(19):2252-7.

39. Salonen JT, Puska P, Kottke TE. Smoking, blood pressure and serum cholesterol as risk factors of acute myocardial infarction and death among men in Eastern Finland. Eur Heart J. 1981;2(5):365-73.

\section{Submit your next manuscript to BioMed Central and we will help you at every step:}

- We accept pre-submission inquiries

- Our selector tool helps you to find the most relevant journal

- We provide round the clock customer support

- Convenient online submission

- Thorough peer review

- Inclusion in PubMed and all major indexing services

- Maximum visibility for your research

Submit your manuscript at www.biomedcentral.com/submit
Biomed Central 